\title{
Multi-camera DIC system with a spatial data stitching procedure for measurements of engineering objects
}

\author{
M. Malesa, K. Malowany and M. Kujawińska \\ Faculty of Mechatronics, Warsaw University of Technology, Boboli 8, 02-525 Warszawa,
}

Received December 23, 2014; accepted December 30, 2014; published December 31, 2014

\begin{abstract}
In this paper we present a multi-camera 3D Digital Image Correlation (DIC) system with the procedure of spatial data stitching. The system diversifies a single DIC setup capability to carry out displacement and strain measurements of big and complex structures such as industrial installations. An exemplary application of a DIC system is described, which concerns the measurements of graded metal plate segments and an error analysis is performed in order to prove practical feasibility of the developed system.
\end{abstract}

The popularity of Digital Image Correlation (DIC) [1] is mostly based on its robustness and ease in adapting to different measurement tasks. Features such as scalable accuracy, scalable field of view, flexibility in selecting the frequency of data acquisition make the DIC feasible for both laboratory and in-situ, industrial applications. However in many cases, the DIC cannot be considered as a real alternative to currently used measurement techniques in industrial practice, due to the trade-off between the maximum dimensions of the field of view and the measurement accuracy and difficulties with measurements of objects with complex shapes where some regions may be obscured or not accessible for DIC due to shades.

Recently, in order to extend DIC measurement capabilities, several groups have been working to develop of multi-camera DIC systems, in which data obtained with individual systems are stitched together in a common coordinate system. According to [2-5] data stitching can be based on different algorithms. The common strategy is to calculate transformation between different 3D DIC setups or individual cameras of multi-camera DIC setup. The transformation in most cases is determined by the analysis of calibration targets viewed simultaneously by individual cameras of a multi-camera DIC setup.

The method for spatial data stitching presented in this paper is tailored so as to satisfy the most important requirements posed by industry, which are flexibility in the selection of field of views and adaptability to different objects, installations or measurement tasks. A general data stitching procedure concerns measurements with a multicamera DIC system consisting of a few 3D DIC setups. Such a system can be used when objects are too big or too complex to be measured with a single DIC setup.
The procedure is based on standard camera calibration [6] and geometrical transformation of individual point clouds gathered in their own coordinate systems into the common coordinate system. The procedure can be applied in the case when 3D DIC setups have overlapping fields of view.

The main goal was to test the applicability and accuracy of a general approach to multi-camera DIC data stitching concerning the measurements of graded metal plate segments. Due to their complex shape and shadings, graded metal plates cannot be measured with a single DIC setup. Furthermore, in the future it is planned to carry out measurements of full-scale self-supporting arch structures with a $12-30 \mathrm{~m}$ span, produced from graded metal plates, which represents relatively big industrial structures. In such an application both issues shadings and size can be overcome by the application of a multi-camera DIC system.

The complex geometry of the metal plates segments (corrugated cross-section) as well as combination of the compression and bending forces make it difficult to numerically model graded metal plates structures. In order to investigate the response of a corrugated cross-section to various loads as well, as to the investigate an impact of cross-section geometry and arch radius on load bearing capacity and buckling mode, one-meter-long metal plates segments have been tested in laboratory conditions. An exemplary metal plates segment (note the numbering of sections) covered with a speckle pattern is presented in Fig. 1a.

The test rig (Fig. 1b) provided a simple support boundary condition and adjustable eccentricity. It also prohibited deplanation of sample ends. Eccentricities can be positive or negative, which simulates the compression of section 1 or section 5 . The results of displacements and strain measurements had to be consequently passed to the finite elements model (FEM) in order to calibrate or optimize the parameters of an orthotropy matrix. Because of the geometry of the samples (Fig. 1a) it was difficult to obtain data accurate enough within the whole sample, using a single DIC system. More specifically, the problem was with observation of sections 2 and 4 of the sample. A single DIC setup observing the sample from the front 
would have difficulties in stereo-correlation in these sections due to shadings. Instead, we used two 3D DIC setups which observed the sample symmetrically from two sides (see Figure 1b). The DIC setup 1 measured sections 1, 2 and 3, while the DIC setup 2 measured sections 3, 4 and 5 . Both systems were purposely set so as to have an overlaid field of view (in section 3). The comparison of the data obtained in this region has been used to assess the accuracy of the data stitching procedure. In both 3D DIC setups PointGrey Grasshopper 5Mpx cameras were used.

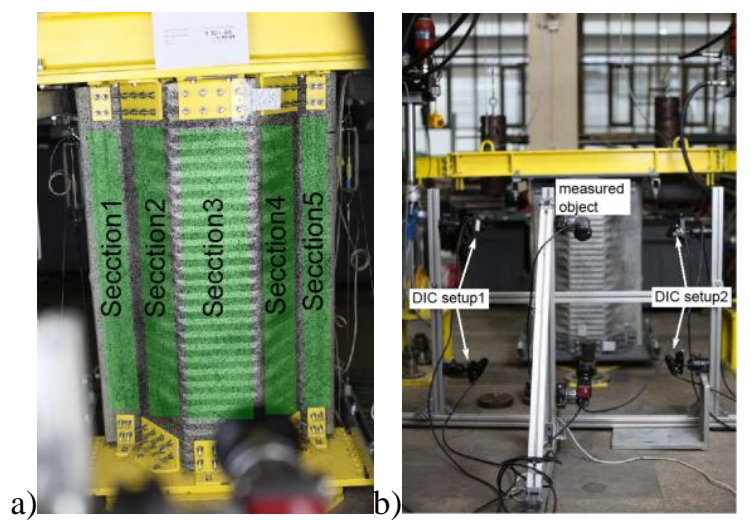

Fig. 1. Measurements of a graded metal plate segment: a) measured metal plates sample in the test rig, b) multi-camera DIC setup.

As both DIC systems had overlaying FOV (Fig. 1) it was possible to use the simplest strategy determining of the $3 \mathrm{D}$ positions of markers used for transformation estimation, which is simultaneous acquisition of images of a calibration artefact (chessboard in this case).

Chessboard corners viewed by each camera have been detected and subsequently used to calculate the 3D position of each marker in coordinate systems of DIC setup 1 and DIC setup 2. The accuracy of determining 3D positions depends strongly on the quality of a standard camera calibration procedure, which has been carried out before the measurement starts. In the presented case, the calibration errors were $0.12 p x$ and $0.14 p x$ respectively for DIC setup 1 and DIC setup 2. The obtained values are relatively big and could have been significantly decreased by setting the lenses and lighting conditions with more care. Relatively poor calibration of DIC setups influenced the next step of the analysis, which was calculating the transformation between coordinate systems of DIC setup 1 and DIC setup 2, with the SVD Method [6].

SVD is a method of matrix factorization into a product of three matrices: unitary matrix $\mathrm{U}$, rectangular, diagonal matrix I and unitary matrix V. Estimating transformation is, in the case of the SVD method, divided into two steps: (i) bringing data to the origin of the coordinate system and then (ii) estimating the translation components $t$ and rotation components $\boldsymbol{R}$.

The transformation parameters obtained in the described system are given in Table 1 .
Table 1. Parameters of the transformation matrix between two DIC setups observing the metal plates.

\begin{tabular}{cccc}
\multicolumn{2}{c}{ Translations $[\mathbf{m m}]$} & \multicolumn{2}{c}{ Rotations $\left[{ }^{\circ}\right]$} \\
$\mathbf{T}_{\mathbf{x}}$ & 56.29 & $\mathbf{R}_{\mathbf{x}}$ & -0.051 \\
$\mathbf{T}_{\mathbf{y}}$ & -794.78 & $\mathbf{R}_{\mathbf{y}}$ & -0.630 \\
$\mathbf{T}_{\mathbf{z}}$ & 172.82 & $\mathbf{R}_{\mathbf{z}}$ & -2.928
\end{tabular}

The transformation error is expressed as a standard deviation of distances between 3D positions of markers (chessboard corners in this case):

$$
T_{\text {error }}=\sqrt{\frac{\sum_{i=1}^{n} \operatorname{dist}\left(T^{*} P 1_{i}, P 2_{i}\right)}{n}},
$$

where $n$ is the number of corners, $P l_{i}$ is the position of a marker in the coordinate system of DIC setup $1, P 2_{i}$ is the position of a marker in the coordinate system of DIC setup 2 and $\boldsymbol{T}$ is the estimated transformation Matrix between both coordinate system.

In the system $\boldsymbol{T}_{\text {error }}$ was determined as $0.098 \mathrm{~mm}$. The areas of interest of both DIC setups covered all segments of the measured metal plates at the analytical stage. Examples of $\mathrm{W}$ displacement maps obtained from both systems separately are presented in Fig. 2.

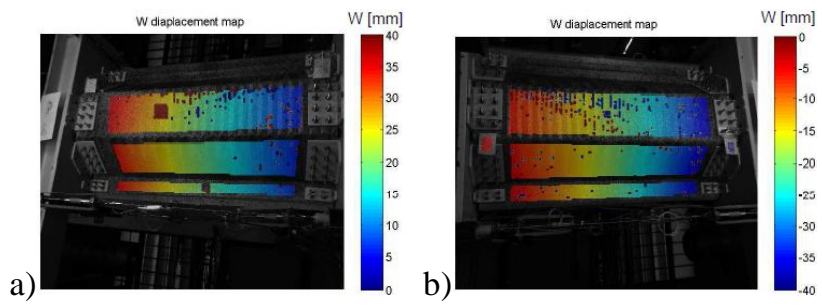

Fig. 2 U displacement maps for low load state obtained from: a) DIC

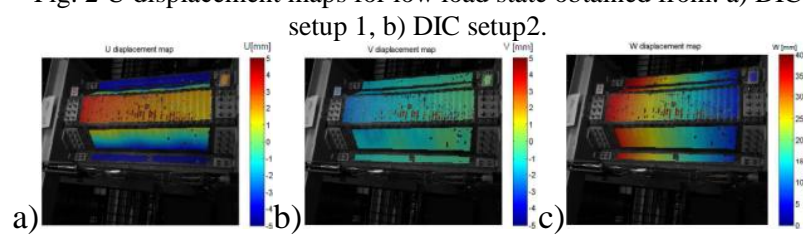

Fig. 3. Displacement maps obtained with both DIC setups after data stitching: a) U displacement map, b) V displacement map, c) W displacement map.

Both DIC systems were unable to correlate data on one of the side walls of the measured object due to shadings (Figs. 2a-b). Combining the data from both systems allowed to fill the missing regions of the area of interest. Final U, V and $\mathrm{W}$ displacement maps, for example of load state, are presented in Figs. 3a-c. The maps presented in Figs. 3 have been obtained by projecting the point cloud from a DIC setup 2 (and transformed to the coordinate system of the DIC setup 1) on the image plane of the reference camera of the DIC setup 1 . The quality of the obtained results could have been improved by performing more accurate calibration procedures of individual 3D DIC setups (i.e. shorter exposure times, bigger depth of focus). A relatively large calibration error influenced the estimation accuracy of 3D positions of chessboard 
corners, which consequently impaired the determination of geometrical transformation between coordinate systems. Also the last step of data analysis, which was the projection of $3 \mathrm{D}$ point clouds on the image plane of the reference camera of the DIC setup 1, was dependent on the calibration quality of the 3D DIC setup 1.

In the next step we evaluated how the obtained transformation error influenced the DIC measurements. This was carried out by calculating discrepancies between $\mathrm{U}, \mathrm{V}$ and $\mathrm{W}$ displacements measured with DIC setup 1 and DIC setup 2 in the middle section of the metal plates (section 3 in Figure 1a). The accepted methodology allowed to calculate discrepancies within a certain areas, not just

in a few points. In this way it was possible to determine if the displacement measurement error, after data stitching, is dependent on the shape of the measured object.

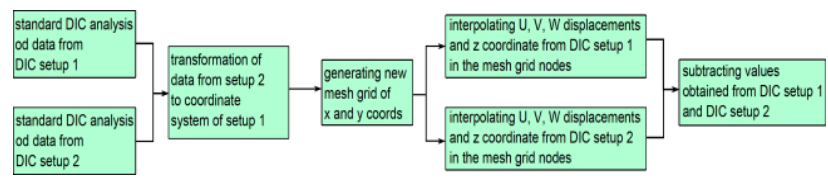

Fig. 4. Data processing path for calculation of discrepancies between DIC setup 1 and DIC setup 2.

In Fig. 4 the data analysis path is presented. In the first step (after data stitching), a new regular grid of $x$ and $y$ coordinates is generated. Then the values of $\mathrm{U}, \mathrm{V}$ and $\mathrm{W}$ displacements as well as the coordinate $z$ have been interpolated to match the $x$ and $y$ coordinates of the new grid. After this procedure the discrepancies have been calculated by simple subtraction between the results obtained from DIC setup 1 and DIC setup 2. The resultant discrepancies maps obtained, for example of a load state, are presented in Fig. 5.
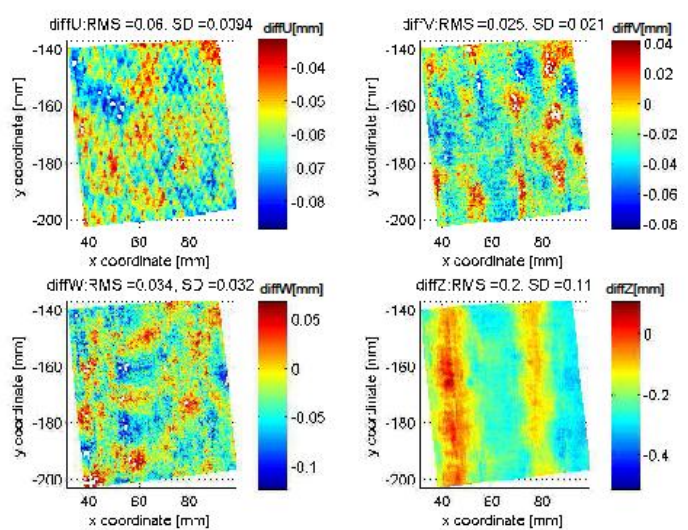

Fig. 3. Discrepancies between U, V, W displacements and z coordinate obtained from DIC setup 1 and DIC setup 2.

The biggest discrepancies occurred for $z$ coordinate ( $R M S=0.2 \mathrm{~mm}$ and $S D=0.11 \mathrm{~mm}$ ). The trends that can be observed in the $z$ coordinate discrepancies maps are correlated with the shape of the measured object. The discrepancies of $W$ displacement were $R M S=0.03 \mathrm{~mm}$ and $S D=0.003 \mathrm{~mm}$. The discrepancies of $\mathrm{U}$ and $\mathrm{V}$ displacements were $(R M S=0.06 \mathrm{~mm}$ and $S D=0.009 \mathrm{~mm})$ and $(R M S=0.025 \mathrm{~mm}$ and $\mathrm{SD}=0.021 \mathrm{~mm})$, respectively. Taking into account the complex shape of the measured object and relatively poor quality of calibration of DIC systems, the obtained values are satisfactory. It is noticeable that they do not increase for the higher load states.

In conclusions, the multi-camera DIC setup has been used to extend the field of view of a standard 3D DIC system in measurements of a metal plates segment and in this way it was possible to present the displacement maps of a relatively complex object in a common coordinate system. The problem with shadings in some regions of the sample has been overcome. The fields of view of individual 3D DIC setups have been purposely set to have overlaying areas of interest. The discrepancies of results obtained in this area after applying the data stitching procedure allowed to assess the accuracy of the procedure. It has been shown that errors are related to the quality of transformation rather than to the load state. Performing more accurate calibration would have reduced the transformation error and provided more precise displacement maps in a multi-camera configuration.

The main task in future research work is to decrease the transformation error in the case of distributed multicamera DIC measurements. Another direction of the future work is to apply much more 3D DIC systems for large engineering objects and to use the method for automatic merging of 3D DIC data distributed in time [7] for temporal stitching of data obtained with multi-DIC systems.

The authors gratefully acknowledge financial support from project OPT4-BLACH financed by the National Center for Research and Development, Marcin Malesa also acknowledge Start Scholarship financed by the Fundation for Polish Science within European Social Funds.

\section{References}

[1] M. Sutton, J.J. Orteu, H. Schreier, Image Correlation for shape, motion and deformation measurements (Springer, 2009).

[2] J.-J. Orteu, F. Bugarin, J. Harvent, L. Robert, V. Velay, Exp. Mechanics 51, 625-639 (2011).

[3] Y. Wang, P. Lava, S. Coppieters, P. V. Houtte, D. Debruyne, Strain 49 , 190-198 (2013).

[4] X. Chen, L. Yang, N. Xua, X. Xie, B. Sia, R. Xu, Optics and Lasers Techn. 57, 318-326 (2014).

[5] C.-H. Hwang, W.-C. Wang, Y.-H. Chen, Proc. SPIE 8769, 876913-1 (2014).

[6] R. Hartley, A. Zisserman, Multiple View Geometry in Computer Vision (Cambridge University Press, 2003).

[7] M. Malesa, M. Kujawińska, Appl. Opt. 52 (19), 4681-4692 (2013). 